\title{
À propos de deux molaires déciduales inférieures provenant des niveaux moustériens de la Grotte du Bison (Arcy-sur-Cure, Yonne, France).
}

Un exemple de lésion cervicale inédite chez un enfant néanderthalien About two lower deciduous molars unearthed from mousterian layers at the Grotte du Bison (Arcy-sur-Cure,Yonne, France) : an intriguing case of cervical tooth lesion in a Neanderthal child

Anne-Marie Tillier, Maurice Hardy, Francine David, Michel Girard et Vladimir d'latchenko

\section{OpenEdition}

\section{Journals}

Édition électronique

URL : http://journals.openedition.org/paleo/2685

DOI : $10.4000 /$ paleo.2685

ISSN : $2101-0420$

Éditeur

SAMRA

Édition imprimée

Date de publication : 15 décembre 2013

Pagination : 271-278

ISSN : $1145-3370$

Référence électronique

Anne-Marie Tillier, Maurice Hardy, Francine David, Michel Girard et Vladimir d'latchenko, «À propos de deux molaires déciduales inférieures provenant des niveaux moustériens de la Grotte du Bison (Arcysur-Cure, Yonne, France). », PALEO [En ligne], 24 | 2013, mis en ligne le 17 avril 2014, consulté le 07 juillet 2020. URL : http://journals.openedition.org/paleo/2685; DOI : https://doi.org/10.4000/paleo. 2685

Ce document a été généré automatiquement le 7 juillet 2020

\section{c)}

PALEO est mis à disposition selon les termes de la licence Creative Commons Attribution - Pas d'Utilisation Commerciale - Pas de Modification 4.0 International. 


\section{À propos de deux molaires déciduales inférieures provenant des niveaux moustériens de la Grotte du Bison (Arcy-sur-Cure, Yonne, France).}

Un exemple de lésion cervicale inédite chez un enfant néanderthalien About two lower deciduous molars unearthed from mousterian layers at the Grotte du Bison (Arcy-sur-Cure,Yonne, France) : an intriguing case of cervical tooth lesion in a Neanderthal child

Anne-Marie Tillier, Maurice Hardy, Francine David, Michel Girard et Vladimir d'latchenko

Nos remerciements s'adressent en premier lieu à François de La Varende pour l'autorisation régulière de conduire des fouilles à la Grotte du Bison. A-m. Tillier remercie Y. Delbos et P. Rouas (Faculté d'Odontologie, Université Bordeaux 2) pour les échanges fructueux qu'ils ont eus. Le manuscrit s'est enrichi suite à une relecture attentive des experts anonymes. Enfin la qualité de l'illustration doit beaucoup à l'amicale contribution de P. Courtaud (UMR 5199-équipe A3P) et G. Devilder (UMR 5133).

\section{Introduction}

Ces dernières années ont vu s'intensifier des études sur la dentition des enfants du Paléolithique, qui ont toutes en commun l'objectif de dégager des différences significatives entre Néanderthaliens et hommes anatomiquement modernes. Ces analyses portent sur des critères morphologiques de la surface occlusale (p.ex. Bailey 2002 ; Bailey and Hublin 2006), sur le temps d'édification des dents déciduales et permanentes (p.ex. Ramirez-Rossi et Bermudez de Castro 2004), ou bien encore sur la 
microstructure des dents et la répartition des tissus dentaires de la couronne (p.ex. Machiarelli et al. 2007 ; Bayle et al. 2009 ; Crèvecoeur et al. 2010).

2 Dans le cas plus spécifique des molaires déciduales inférieures, plusieurs critères ayant trait au contour de la couronne en vue occlusale (forme ovoïde avec rapprochement bucco-lingual des cuspides) et à la structure de la surface occlusale elle-même, ont été avancés pour caractériser les dents néanderthaliennes, tels qu'un développement de la fovéa antérieure, la présence d'une crête d'émail continue reliant le protoconide au métaconide, (« substantial mid-trigonid crest » selon Bailey et Hublin 2006 - p. 505).

Cependant, la présence sur la seconde molaire néanderthalienne d'une crête continue ne semble pas un caractère constant, comme en témoignent, par exemple en France, les fossiles de Chateauneuf 2 (Tillier 1979), Roc de Marsal (Madre-Dupouy 1992, observation pers.) ou bien encore La Chaise 13 et 14 (Tillier et Genet-Varcin 1980). Chez ces derniers, un sillon mésio-distal divise cette crête transversale, une configuration qui se retrouve sur d'autres dents du Paléolithique moyen, telles celles des premiers enfants modernes de Qafzeh (Tillier 1999).

4 L'étude des deux molaires déciduales mises au jour à la Grotte du Bison doit permettre de les positionner dans la variabilité connue et de déceler la présence éventuelle d'anomalies ou de traits pathologiques.

\section{Historique et contexte archéologique}

Les Grottes d'Arcy-sur-Cure (Yonne) ont fait l'objet de nombreux travaux depuis le 19e siècle qui ont livré d'importants documents archéologiques et anthropologiques relevant du Paléolithique supérieur et moyen, comme de la transition entre ces deux périodes (p.ex. Leroi-Gourhan A. 1950, 1958, 1961; Leroi-Gourhan Arl. et A. 1964 ; Baffier et Girard 1997; David et al. 2007, 2009). Dans la Grotte du Bison, des fouilles programmées ont permis depuis 1995 la reconnaissance de plusieurs niveaux (fig. 1) d'occupation humaine, du Châtelperronien final (niveau D, daté entre $34050 \pm 750$ et $33670 \pm 450$ ans avant le présent) au Moustérien avec les couches $\mathrm{E}$ à $\mathrm{J}$ (David et al. 2007, 2009). Dans les couches $\mathrm{E}$ et $\mathrm{F}$, il s'agit d'un Moustérien «final » à débitage Levallois (daté entre $38400 \pm 1600$ et $40200 \pm 1500$ ans avant le présent) avec des structures d'habitat et foyers, tandis que les couches $\mathrm{G}$ et $\mathrm{H}$ ont livré un Moustérien à denticulés qui peut être attribué au stade isotopique OIS 4 (David et al.op.cit.). Ces deux dernières couches, comme la couche I sous-jacente rattachable à l'horizon moustérien de base (Lhomme in David et al. 2007), n'ont pas encore fait l'objet de datations absolues. 
Figure 1 - La Grotte du Bison : profil stratigraphique transversal de la partie antérieure redessiné par G. Devilder indiquant la position de la couche I dont proviennent les dents

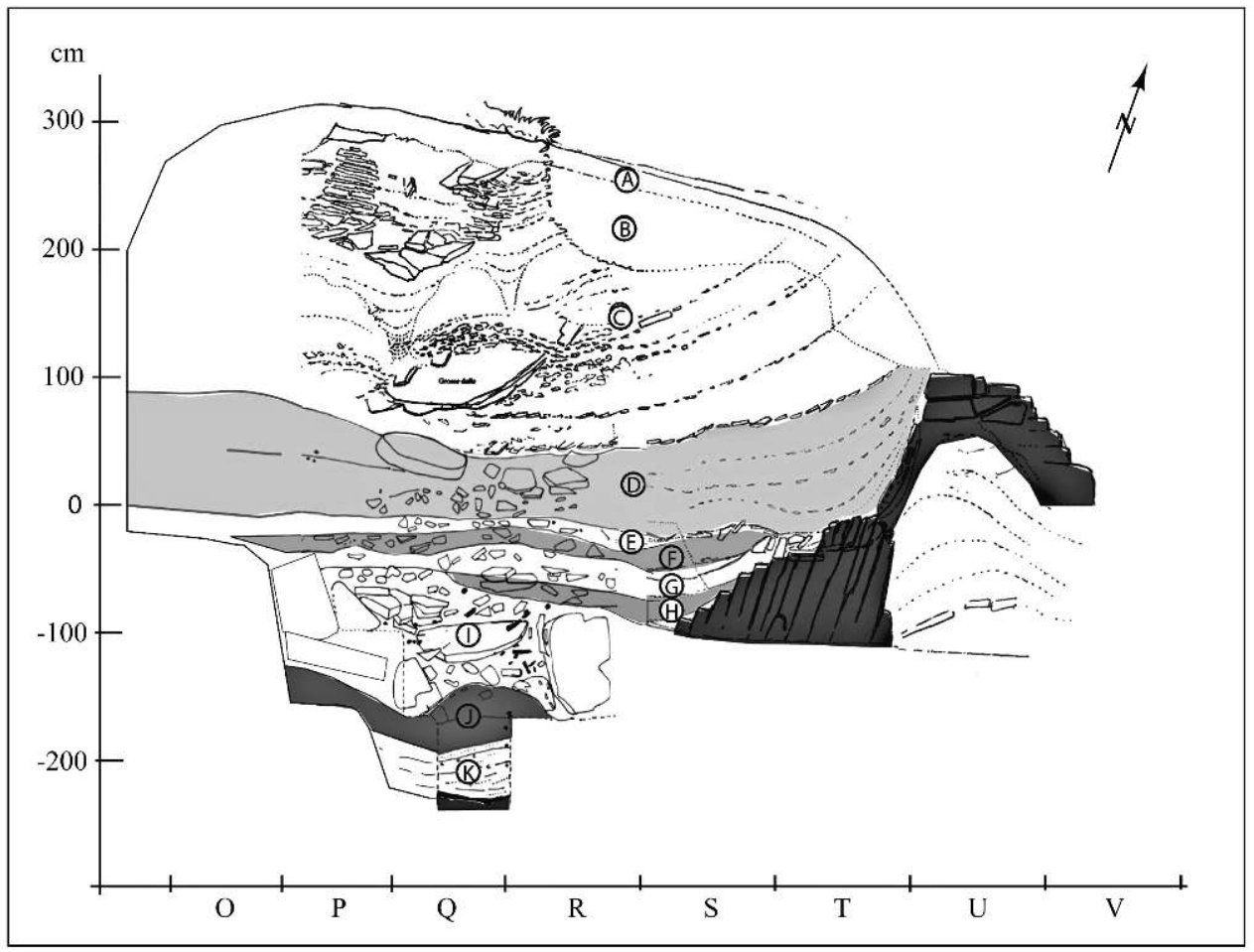

6 La couche I a livré ces dernières années plusieurs vestiges humains fragmentaires, dont certains portaient des traces de l'activité des carnivores qui ont fréquenté régulièrement la grotte (David et al. 2009; Tillier et al. 2013). Deux nouvelles dents humaines (BIS-I S6-2 et BIS I Q5.1), mises au jour en 2012, (Hardy et al. 2012) viennent compléter la documentation anthropologique déjà publiée. Elles sont comparées aux autres dents moustériennes déjà connues en Europe, ainsi qu’à la seule dent déciduale de même rang (spécimen 29 issu de la couche Xb2) qui provient du niveau Châtelperronien de la Grotte du Renne à Arcy sur-Cure (Bailey et Hublin 2006).

\section{Estimation de l'âge au décès des enfants représentés par BIS-I S6-2 et BIS I Q5.1.}

7 La dent BIS-I S6-2 est une seconde molaire déciduale inférieure droite (fig. 2). La couronne est complète et l'édification des racines (fig. 2a) correspond au stade R3/4 défini par Moorrees et al. (1963), ou au stade F de Liversidge et Molleson (2004). Les faces mésiale et distale ne présentent aucune facette de contact avec les dents adjacentes. L'âge de l'enfant auquel appartenait cette dent, selon les critères de référence issus de l'étude des populations actuelles, peut être estimé à $3.12 \pm 0.26$ ans, selon les formules de Liversidge et Molleson (2004). 
Figure 2 - La seconde molaire déciduale inférieure droite (BIS-I S6-2) de la Grotte du Bison en vues buccale (a) et occlusale (b)
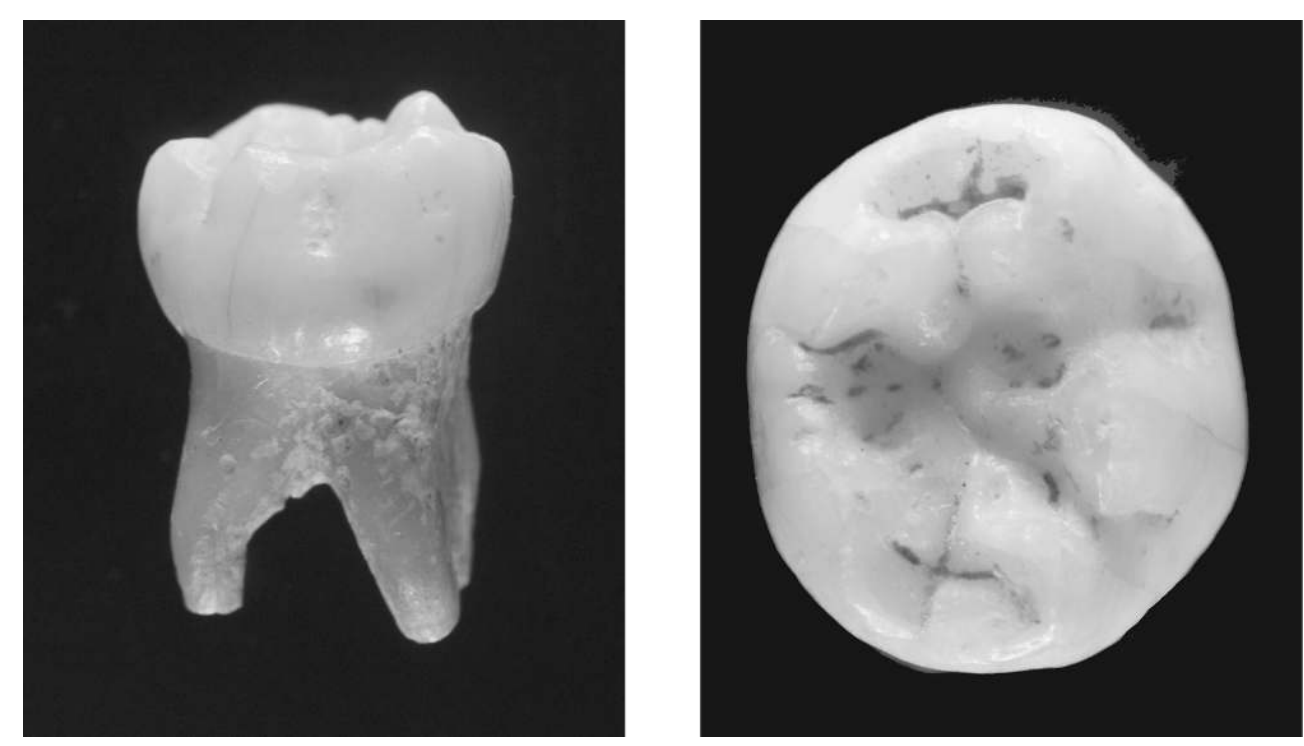

8 La dent BIS I Q5.1 est également une seconde molaire déciduale inférieure droite (fig. 3). Les deux racines mésiale et distale ont débuté leur résorption, une résorption plus avancée pour la première (fig. 3a). Le degré d'attrition de la surface occlusale s'accompagne d'une mise à nu de la dentine sous forme de lentilles à l'emplacement des cuspides. La présence d'une facette de contact nette sur la face distale de la couronne atteste que la première molaire permanente était fonctionnelle. L'âge de l'enfant auquel a appartenu cette dent, selon les données des populations actuelles, peut être envisagé entre 8 et 10 ans. 
Figure 3 - La seconde molaire déciduale inférieure droite (BIS I Q5.1) de la Grotte du Bison en vues buccale (a), occlusale (b) et mésiale (c).
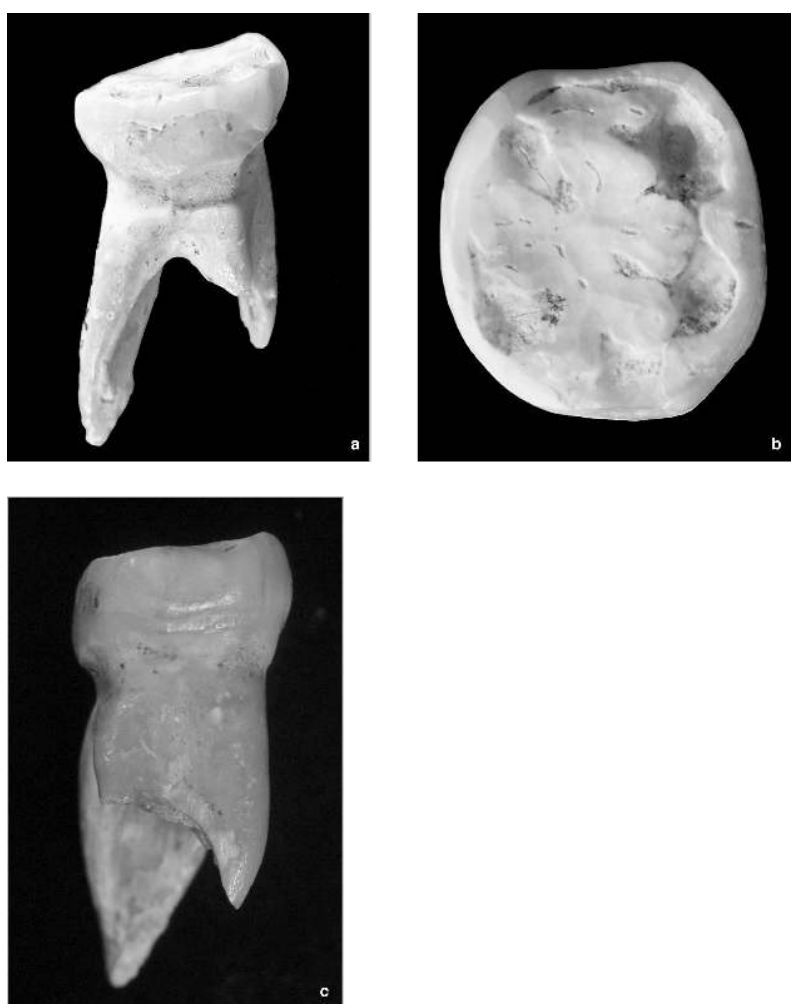

Les nouvelles molaires déciduales inférieures de la Grotte du Bison, sont deux dents droites, ce qui exclut leur appartenance à un même enfant; par ailleurs, leur stade d'édification atteste une appartenance à deux enfants distincts.

\section{Caractères morphologiques des molaires déciduales}

\section{1 - BIS-I S6}

La couronne de la dent BIS-I S6 s'inscrit dans un rectangle et la configuration de la surface occlusale est bien identifiable (fig. $2 \mathrm{~b}$ ) : il s'agit d'une configuration classique à cinq cuspides, trois buccales et deux linguales, dont la distribution ne témoigne d'aucun resserrement bucco-lingual. L'ordre de taille décroissant de ces cuspides est le suivant : protoconide et métaconide, hypoconide et entoconide, hypoconulide. Les deux cuspides mésiales, à savoir le protoconide et le métaconide, sont reliées entre elles par une crête dont le trajet est interrompu en son milieu par un petit sillon sagittal ; cette crête délimite une fovéa antérieure étroite. La fovéa centrale est vaste et on note la présence de deux sillons descendant sur la face buccale qui séparent les cuspides buccales les unes des autres. Si le protoconide et le métaconide sont alignés mésiodistalement, un décrochement s'observe avec la cuspide bucco-distale plus réduite et décalée lingualement. 


\section{2 - BIS I Q5.1}

11 La couronne de la seconde molaire déciduale, BIS I Q5.1, du fait de l'usure de la dent, s'inscrit globalement dans un carré (fig. 3b). Compte tenu du développement d'une facette de contact sur la face distale, l'emplacement de la cuspide bucco-distale (hypoconulide) est de fait estompé. Quatre lentilles de dentine, deux alignées mésiodistalement et deux bucco-lingualement, sont présentes et correspondent aux quatre cuspides principales dont le degré d'usure est quasi-équivalent. Aucun relief n'est discernable sur la surface occlusale, la fovéa centrale étant elle aussi érodée.

\section{3 - Données comparatives}

Du point de vue morphologique, la surface occlusale des deux molaires déciduales de la Grotte du Bison, par le nombre des cuspides et la présence d'une crête transversale délimitant la fovéa antérieure, ne se distingue pas fondamentalement de celles des autres dents identifiées comme néanderthaliennes du Paléolithique européen (moyen et supérieur). La disposition de la crête transversale nette sur la molaire non usée BIS-I S6 montre un trajet discontinu qui évoque la disposition d'autres dents néanderthaliennes (tels Roc de Marsal, La Chaise 13 et 14, Chateauneuf 2 par exemple), s'écartant ainsi de celle mentionnée comme la plus fréquente dans la population néanderthalienne (Bailey et Hublin 2006).

13 Une comparaison avec la molaire de la Grotte du Renne (29-Xb2 C8) permet de dégager quelques traits supplémentaires qui distinguent la dent de la Grotte du Bison : cette dernière ne présente pas de rapprochement bucco-lingual des cuspides (décrit sur la dent 29-Xb2 C8 par Bailey et Hublin 2006). Sur la molaire de la Grotte du Bison, la cuspide bucco-distale (hypoconulide) seule est en positionnement plus interne et sa taille est plus réduite que sur la dent de comparaison ; il n'y a pas de sixième cuspide et enfin la surface occlusale est moins subdivisée secondairement. Par ce dernier trait, la dent s'écarte également de la molaire châtelperronienne de rang équivalent de la Grotte du Renne qui provient d'un enfant plus jeune âgé de 7-15 mois (selon Bailey et Hublin 2006); sur cette dernière, des sillons secondaires complexifiant la surface occlusale sont observables.

\section{Dimensions coronaires et comparaison}

Les dimensions coronaires des deux secondes molaires déciduales inférieures de la Grotte du Bison, ainsi que le montre le tableau 1, s'intègrent dans la variation néanderthalienne connue (quelle que soit la période considérée) comme dans celle des dents du Paléolithique supérieur. On peut noter que les couronnes sont toutes deux assez étroites bucco-lingualement, se rapprochant par ce trait de la dent châtelperronienne de la Grotte du Renne (29-Xb2 C8). La légère différence de taille relevée entre les deux dents de la Grotte du Bison pour le diamètre mésio-distal peut s'expliquer par la présence d'une facette d'usure sur la face distale de la molaire BIS-IQ5-1. 
Tableau 1 - Dimensions coronaires (en $\mathrm{mm}$ ) des secondes molaires déciduales inférieures de la Grotte du Bison (MD : longueur mésio-distale ; BL : largeur bucco-linguale), comparées aux dents du Paléolithique européen. Sources bibliographiques (1) Bailey et Hublin 2006, (2) Tillier et al. 2003, (3) Hillson et Trinkaus 2002, (4) Benazzi et al. 2010, (*) échantillon regroupe des enfants du Paléolithique supérieur et actuels

\begin{tabular}{|l|l|l|}
\hline & $\mathrm{MD}$ & $\mathrm{BL}$ \\
\hline Grotte du Bison I S6 & 10.0 & 8.7 \\
\hline Grotte du Bison I Q5.1 & 9.5 & 8.6 \\
\hline $\begin{array}{l}\text { Grotte du Renne 29 } \\
\text { Xb2 C8 droite (1) } \\
\text { Châtelperronien }\end{array}$ & 10.0 & 8.9 \\
\hline $\begin{array}{l}\text { Néanderthaliens (2) } \\
\text { Moustérien }\end{array}$ & $\begin{array}{l}10.3 \pm 0.6 \\
(\mathrm{~N}=15)\end{array}$ & $\begin{array}{l}9.3 \pm 0.4 \\
(\mathrm{~N}=15)\end{array}$ \\
\hline $\begin{array}{l}\text { Enfants modernes } \\
\text { Paléolithique supérieur (3) }\end{array}$ & $\begin{array}{l}10.3 \pm 0.7 \\
(\mathrm{~N}=5)\end{array}$ & $\begin{array}{l}9.3 \pm 0.7 \\
(\mathrm{~N}=13)\end{array}$ \\
\hline $\begin{array}{l}\text { Enfants modernes* } \\
(\mathrm{N}=18) \text { (4) }\end{array}$ & $\begin{array}{l}\text { moyenne = 9.93 } \\
\text { écart-type =0.51 }\end{array}$ & $\begin{array}{l}\text { moyenne écart-type = }=0.42 \\
\text { éco }\end{array}$
\end{tabular}

\section{Aspects paléopathologiques et anomalies}

\section{1 - Lésions carieuses, hypoplasies de l'émail}

Selon la littérature spécialisée, la fréquence de lésions carieuses serait limitée sur les dents du Paléolithique moyen d'Eurasie (Sognnaes 1956; Boydstun, Trinkaus, Vandermeersch 1988; Tillier et al. 1995; Trinkaus, Smith, Lebel 2000 ; Trinkaus et Pinilla 2009; Tillier et Sansilbano-Collilieux 2010). Prenant en considération la distribution géographique des fossiles affectés, qui se répartissent sur le pourtour méditerranéen (sud-est de la France et Israël), des auteurs (Trinkaus et Pinilla, ibidem) ont suggéré de mettre en relation la présence de caries avec une alimentation plus riche en hydrates de carbone, liée à la composition de la faune, ce que les auteurs mentionnaient comme "general absence of cold climate fauna in the archaeological assemblage » (2009, p. 74). A notre connaissance, pour l'Europe et les Néanderthaliens, il n'existerait qu'une molaire déciduale venant du Bau de l'Aubesier porteuse de carie (Trinkaus et al. 2000). Les nouvelles molaires déciduales de la Grotte du Bison, tout comme celles précédemment décrites à la Grotte du Renne (Bailey et Hublin 2006) sont exemptes de lésion carieuse.

Les dents peuvent également porter des hypoplasies linéaires de l'émail sur la surface externe de la couronne, considérées comme des indicateurs de stress non spécifiques dont l'étiologie reste assez débattue parmi les auteurs (p.ex. Goodman et Rose 1990; Neiburger 1990; Schultz et al. 1998), notamment quant au rôle éventuel de carences nutritionnelles. Les investigations conduites sur des fossiles du Pléistocène supérieur d'Europe quant à la présence d'hypoplasies de l'émail (au-delà des seules hypoplasies linéaires) rendent compte de fréquences variables selon les auteurs (p.ex. Ogilvie et al. 1989 ; Brennan 1991 ; Berti et Mahaney 1992 ; Garcia Sanchez et al. 1994 ; Skinner 1996 ; Guatelli-Stenberg et al. 2004), en fonction de la nature des hypoplasies prises en compte. Un faible pourcentage incluant tous types d'hypoplasies a été relevé pour les dents déciduales néanderthaliennes $(3,3 \%)$. Aucune des deux nouvelles dents de La Grotte du Bison ne présente d'hypoplasie de l'émail, quelle que soit la catégorie considérée. Ce constat rejoint celui déjà formulé pour les autres dents déciduales des 
deux Grottes d'Arcy-sur-Cure, la Grotte du Renne (Bailey et Hublin 2006) et la Grotte du Bison (Tillier et al. 2013).

\section{2 - Lésion cervicale affectant la molaire BIS I Q5.1}

17 La dent BIS I Q5.1 se distingue par la présence d'une perte de substance qui affecte la région cervicale le long de la face buccale et très partiellement aussi la face mésiale (fig. 3a et c). Cette lésion prend l'aspect d'un sillon élargi dont le fond est lisse. Le décrochement qui en résulte à la hauteur du collet est bien visible sur une vue mésiale de la dent (fig. 3c). Ce type de lésion d'abrasion n'a jamais été décrit sur les dents déciduales néanderthaliennes. Peut-on assimiler cette altération de surface à une mylolyse ? Et quelle en serait la cause?

Dans la littérature spécialisée, la présence de lésions d'abrasion au niveau du collet (parmi lesquelles les Non carious Cervical Tooth Lesions ou encore NCCLs) n'est décrite, dans les populations actuelles, que sur les dents permanentes. N'ayant pas une origine bactérienne, elles présenteraient différentes formes selon leur degré d'atteinte, une dépression étroite, une lésion sphérique ou une atteinte abrupte en forme de coin. Leur étiologie semble encore mal cernée (p.ex. Levitch et al. 1994 ; Grippo, Simring, Coleman 2012): des facteurs chimiques d'origines diverses, des contraintes biomécaniques dépendant d'une éventuelle maloclusion (liée notamment à une "tooth flexure » ou angulation de la dent), un brossage intensif des dents, un stress occlusal, éventuellement le bruxisme, font partie des causes invoquées dans l'apparition de ces lésions. Une absence de NCCLs relevée dans des collections archéologiques de l'Âge du Cuivre et du Moyen-Âge du sud de la France a été mise en relation avec le mode d'alimentation, le mode de vie (absence de nettoyage intensif des dents par exemple), voire une constitution dentaire différente (Aubry et al. 2003).

En conclusion, la modification de surface observée sur la molaire BIS I Q5.1 de la Grotte $\mathrm{du}$ Bison serait unique puisqu'affectant une dent de la première dentition. Elle constituerait de plus une exception parmi les dents néanderthaliennes déciduales. En l'absence de tout élément de comparaison, son interprétation demeure problématique, même si toute origine strictement taphonomique est à exclure. L'étendue de la dépression sur la face buccale de la région cervicale de la dent ainsi que son aspect régulier et lisse inciteraient à privilégier un exemple de lésion cervicale non carieuse, bien qu'aucun cas de ce type de lésion n'ait été signalé sur la première dentition. Cependant, une cause d'origine biomécanique, ou une activité spécifique durant la vie du sujet, peuvent-elles être compatibles avec le jeune âge au décès de l'enfant, entre 8 et 10 ans? L'étiologie de cette lésion identifiée pour la première fois sur une dent déciduale, et qui plus est néanderthalienne, soulève donc à double titre, bien des interrogations. 


\section{BIBLIOGRAPHIE}

AUBRY M., MAFART B., DONAT B., BRAU J.J. 2003 - Brief Communication. Study of Non Carious Cervical Tooth lesions in samples of Prehistoric, Historic and Modern Populations from the South of France. American Journal of Physical Anthropology, 121, p. 10-14.

BAFFIER D., GIRARD M. 1997 - Le karst d'Arcy-sur-Cure (Yonne) et ses occupations humaines, Quartenaire, v. 8, p. 245-255.

BAILEY S.E. 2002 - Neandertal Dental Morphology : implications for Modern Human Origins. PhD Dissertaion, Arizona Sate University, 238 p.

BAILEY S.E., HUBLIN J-J. 2006 - Dental remains from the Grotte du Renne at Arcy-sur-Cure (Yonne). Journal of Human Evolution, 50, p. 485-508.

BAYLE P., BRAGA J., MAZURIER A., MACCHIARELLI R. 2009 - Dental development pattern of the Neanderthal child from Roc de Marsal : a high-resolution 3D analysis. Journal of Human Evolution, 56, p. 66-75.

BENAZZI S., FORNAI C., BAYLE P., COQUERELLE M., KULLMER O., MALLEGNI F., WEBER G.W. 2011 Comparison of dental measurement systems for taxonomic assignment of Neanderthal and modern human lower deciduous molars. Journal of Human Evolution, 61, p. 320-326.

BERTI PR., MAHANEY MC. 1992 - Quantification of the confidence interval of linear enamel hypoplasia chronologies. In : LL. Capasso \& AH. Goodman (Eds) Recent Contributions to the study of Enamel Developmental defects. Joural of Paleopathology Monogr. Publ.2, Chieti (Italy), p. 19-30.

BOYDSTUN S. B., TRINKAUS E., VANDERMEERSCH B. 1988 - Dental caries in the Qafzeh 3 early modern human. American Journal of Physical Anthropology, 75, abstract, p. 188-189.

BRENNAN M.U. 1991 - Health and disease in the Middle and Upper paleolithic of Southwestern France. PhD Dissertation, New York University.

CRÈVECOEUR I., BAYLE P., ROUGIER H., MAUREILLE B., HIGHAM T., VAN DER PLICHT J., DE CLERK N., SEMAL P. 2010 - The Spy VI child : a newly discovered Neandertal infant. Journal of Human Evolution, 59, p. 641-656.

DAVID F., D'IATCHENKO V.I., ENLOE J.E., GIRARD M., HARDY M., LHOMME V., ROBLIN-JOUVE A. 2007 - Arcy-sur-Cure (Yonne) - Grotte du Bison ; Compte-rendu des travaux 2005-2007, Dijon, Service Régional de l'Archéologie de Bourgogne, novembre 2007, 60 p.

DAVID F., D'IATCHENKO V.I., ENLOE J.E., GIRARD M., HARDY M., LHOMME V., ROBIN-JOUVE A., TILLIER A.-M, TOLMIE C. 2009 - New Neandertal remains from the Grotte du Bison at Arcy-surCure, France. Journal of Human Evolution, 57, p. 805-809.

GARCIA SANCHEZ M., TILLIER A.-m, GARRALDA MD. VEGA-TOSCANO G. 1994 - Les dents d'enfant des niveaux moustériens de la Grotte de Carihuela (Grenade, Espagne). Paleo, 6, p. 79-88.

GOODMAN A.H., ROSE J.C.1990 - Dental enamel hypoplasias as indicators of nutritional status. Yearbook of Physical Anthropology, New York, Wiley Liss, p. 279- 293.

GRIPPO J.O., SIMRING M., COLEMAN T.A. 2012 - Abfraction, Abrasion, Biocorrosion, and the Enigma of Non carious Cervical Lesions : a 20-year Perspective. Journal of Esthetic and Restorative Dentistry, 24, 1, p. 10-23. 
GUATELLI-STENBERG, D., LARSEN, CS., HUTCHINSON, DL. 2004 - Prevalence of the duration of Linear enamel hypoplasia : a comparative study of Neandertals and Inuit foragers. Journal of Human Evolution, 47, p. 65-84.

HARDY M., DAVID F., d'IATCHENKO V., GIRARD M., ROBLIN-JOUVE A. 2012 - Arcy-sur-Cure (Yonne). Grotte du Bison. Comptes Rendus des travaux 2012. Service Régional de l'Archéologie de Bourgogne, Dijon, $91 \mathrm{P}$.

HILLSON S.W., TRINKAUS E. 2002 - Comparative Dental Crown Metrics. In ; J. Zilhao and E. Trinkaus, (Eds), Portrait of the Artist as a Child. The Gravettian Human Skeleton from the Abrigo do Lagar Velho and its archaeological context. Trabalhos de Arqueologia 22, Lisboa, p. 356-364.

LEROI-GOURHAN A. 1950 - La Grotte du Loup, Arcy-sur-Cure (Yonne). Bulletin de la Société Préhistorique Française, t. XXXXVII, n 5, p. 268-280.

LEROI-GOURHAN A. 1958 - Étude des restes humains fossiles provenant des Grottes d'Arcy-surCure. Annales de Paléontologie, 44, p. 87-148.

LEROI-GOURHAN A. 1961 - Les fouilles d'Arcy-sur-Cure (Yonne), Gallia Préhistoire, t. IV, p. 3-16.

LEROI-GOURHAN Arl., LEROI-GOURHAN A. 1964 - Chronologie des Grottes d'Arcy-sur-Cure (Yonne). Gallia Préhistoire, t. 7, p. 1-64.

LEVITCH L.C., BADER J.D., SHUGARS D.A., HEYMANN H.O. 1994 - Non carious cervical lesions. Journal of Dentistry, 22, p. 195-207.

LIVERSIDGE H.M., MOLLESON T. 2004 - Variation in Crown and Root Formation and Eruption of Human Deciduous Teeth. American Journal of Physical Anthropology, 123, p. 172-180.

MACHIARELLI R., MAZURIER A., VOLPATO V. 2007 - L'apport de nouvelles technologies à l'étude des Néandertaliens. In : B. Vandermeersch, B. Maureille (Eds.), Les Néandertaliens. Biologie et Cultures. Editions du C.T.H.S., Paris, p. 169-179.

MADRE-DUPOUY M. 1992 - L'enfant du Roc de Marsal. Etude analytique et comparative. Cahiers de Paléoanthropologie, Editions du CNRS, Paris, 300 p.

MOORREES C.F.A., FANNING E. A. \& HUNT E. E.1963 - Formation and Resorption stages of Three Deciduous Teeth in Children. American Journal of Physical Anthropology. 21, p. 99-108.

NEIBURGER E. 1990 - Enamel hypoplasias : poor indications of dietary stress. American Journal of Physical Anthropology, 82, p. 231-232.

OGILVIE M. D., CURRAN B. K., TRINKAUS E. 1989 - The incidence and patterning of dental enamel hypoplasias among the Neandertals. American Journal of Physical Anthropology, 79, p. 25-41.

RAMIREZ-ROSSI F.V., BERMUDEZ DE CASTRO J.M. 2004 - Surprisingly rapid growth in Neandertal. Nature, 428, p. 936-939.

SCHULTZ M., CARLI-THIELE P., SCHMIDT-SCHULTZ T.H., KIEDORF U., KIEDORF H., TEEGEN W-R.. KREUT K. 1998 - Enamel Hypoplasias in Archaeological Skeletal remains. In : KW. Alt, FW. Rösing \& M. Tescher-Nicola (Eds.). Dental Anthropology. Fundamentals, Limits and Propects. Springer Wien New York, p. 293-312.

SKINNER M. 1996 - Developmental Stress in Immature Hominines from Late Pleistocene Eurasia : Evidence from enamel Hypoplasia. Journal of Archaeological Science, 23, p. 833852.

SOGNNAES R.F. 1956 - Histological Evidence of Developmental Lesions in Teeth originating from Paleolithic, Prehistoric and Ancient Man. American Journal of Pathology, 32, p. 547-577. 
TILLIER A.-m 1979 - La dentition de l'enfant moustérien Châteauneuf 2 découvert à Hauteroche (Charente). L'Anthropologie, 83/3 ? 417-438.

TILLIER A.-m 1999 - Les enfants moustériens de Qafzeh. Interprétation phylogénétique et paléoauxologique. Cahiers de Paléoanthropologie, CNRS Editions, Paris.

TILLIER A.-m, ARENSBURG B., RAK Y., VANDERMEERSCH B. 1995 - Middle Palaeolithic dental caries : new evidence from Kebara (Mount Carmel, Israel). Journal of Human Evolution, 29, p. $189-192$.

TILLIER A.-m, ARENSBURG B., VANDERMEERSCH B., CHECH M. 2003 - New Human remains from Kebara Cave (Mount Carmel). The place of the Kebara hominids in the Levantine Mousterian Fossil Record. Paléorient, 29- 2, p. 35- 62.

TILLIER A.-m, GENET-VARCIN E. 1980 - La plus ancienne mandibule d'enfant découverte dans le gisement de La Chaise de Vouthon (Abri Suard), Charente, France. Zeischrift. fur Morpholologie und Anthropologie, 71/2, p. 196-214.

TILLIER A.-m, SANSILBANO-COLLILIEUX M. 2010 - Early Hunter-gatherers and Oral health. Inferences from Middle Palaeolithic hominids in Southern Levant. 7ICAANE, London, Programme and Abstract Handbook, p. 157.

TILLIER A.-m, SANSILBANO-COLLILIEUX M., DAVID F., ENLOE J.G., GIRARD M., HARDY M., D'IATCHENKO V., ROBLIN-JOUVE A., TOLMIE C. 2013 - Les vestiges néanderthaliens provenant des niveaux moustériens de la Grotte du Bison à Arcy-sur-Cure (Yonne). Bulletins et Mémoires de la Société d'Anthropologie de Paris, 1-2, p. 39-54.

TRINKAUS E., SMITH R.J., LEBEL S. 2000 - Dental caries in the Aubesier 5 Neandertal Primary Molar. Journal of Archaeological Science, 27, p. 1017-1021.

TRINKAUS E., PINILLA B. 2009 - Dental Caries in the Qafzeh 3 Middle Paleolithic Modern Human. Paléorient, 36/1, p. 69-76.

\section{RÉSUMÉS}

La Grotte du Bison (Arcy-sur-Cure, Yonne) a livré une séquence de sept niveaux d'occupation humaine allant du Paléolithique moyen (Moustérien) au début du Paléolithique supérieur (Châtelperronien). Des fouilles récentes ont permis la mise au jour de quelques vestiges humains néanderthaliens dans les niveaux moustériens J et I (David et al. 2007 ; Tillier et al. 2013). Lors de la campagne de 2012, deux molaires déciduales inférieures ont été découvertes dans la couche I, apportant de nouvelles informations sur les derniers artisans des industries du Paléolithique moyen dans le nord-est de la France. L'une des dents présente une lésion cervicale non carieuse de l'émail, inédite chez l'enfant, ce qui pose la question de son étiologie.

At the Grotte du Bison (Arcy-sur-Cure, Yonne), the geological sequence includes seven levels of human occupations from the Mousterian (Middle Palaeolithic) to the Chatelperronian (Early Upper Palaeolithic). Recent excavations have led to the discovery of few neanderthal human remains in Mousterian layers J and I (David et al. 2007; Tillier et al. 2013). Two additional deciduous lower molars were found in layer I during the 2012's season. These new anthropological finds add to our knowledge about the authorship of final Middle Palaeolithic industries in north-eastern France. One of the teeth shows a non-carious cervical lesion, unknown in children, which raises the question of its etiology. 
INDEX

Keywords : Arcy-sur-Cure, Grotte du Bison, Mousterian, Neanderthal, deciduous tooth, noncarious cervical lesion

Mots-clés : Arcy-sur-Cure, Grotte du Bison, Moustérien, Néanderthal, dent déciduale, lésion cervicale non carieuse

\section{AUTEURS}

\section{ANNE-MARIE TILLIER}

Université Bordeaux 1, UMR 5199 PACEA, Anthropologie des Populations Passées et Présentes, avenue des Facultés, FR-33405 Talence - am.tillier@pacea.u-bordeaux1.fr

\section{MAURICE HARDY}

UMR 7041, ArScAn, Laboratoire d'Ethnologie préhistorique, Maison René Ginouvès, 21 allée de l'Université, FR- 92023 Nanterre Cedex.

\section{FRANCINE DAVID}

UMR 7041, ArScAn, Laboratoire d'Ethnologie préhistorique, Maison René Ginouvès, 21 allée de l'Université, FR- 92023 Nanterre Cedex.

\section{MICHEL GIRARD}

342, Boulevard des Horizons, FR-06220 Vallauris

\section{VLADIMIR D'IATCHENKO}

Musei Anthropologii i Etnografii, Kunstkamera, Saint-Petersburg, Russie. 